\title{
Small Gröbner Fans of Ideals of Points
}

\author{
Elena Dimitrova ${ }^{1}$ \\ Department of Mathematical Sciences, Clemson University, Clemson, SC 29634, USA \\ Qijun $\mathrm{He}^{1}$ \\ Biocomplexity Institute and Initiative, Charlottesville, VA 22911, USA \\ Lorenzo Robbiano \\ Dip. di Matematica, Università degli Studi di Genova, Via Dodecaneso 35, I-16146 Genova, \\ Italy \\ Brandilyn Stigler ${ }^{1}$ \\ Department of Mathematics, Southern Methodist University, Dallas, TX 75275, USA
}

\begin{abstract}
In the context of modeling biological systems, it is of interest to generate ideals of points with a unique reduced Gröbner basis, and the first main goal of this paper is to identify classes of ideals in polynomial rings which share this property. Moreover, we provide methodologies for constructing such ideals. We then relax the condition of uniqueness. The second and most relevant topic discussed here is to consider and identify pairs of ideals with the same number of reduced Gröbner bases, that is, with the same cardinality of their associated Gröbner fan.
\end{abstract}

Keywords: Ideal of points, basic set, staircases, Gröbner fan, distraction, complementary ideals.

2000 MSC: 2010 13P10, 13P25, 13-04, 68W30, 92B05

\section{Introduction}

Gröbner bases have enjoyed a diverse set of applications since their inception in 1965 (for example, see [12, 13, 16, 17]). In 2004, Gröbner bases were applied to the problem of model selection in systems biology [11]. Specifically, they were introduced as a tool to select minimal models from a set of polynomial dynamical systems (PDS) that fit discretized experimental data: for a given set of data points over a finite field, the ideal of points forms a coset representing

Email addresses: edimit@clemson.edu (Elena Dimitrova), qh4nj@virginia.edu (Qijun

He), robbiano@dima.unige.it (Lorenzo Robbiano), bstigler@smu.edu (Brandilyn Stigler)

${ }^{1}$ Partially supported by NSF grant DMS-1419023. 
the space of PDSs that fit the data and a minimal model is selected from the space by computing a reduced Gröbner basis of the ideal and taking the normal forms of the model equations. While this provides an algorithmic solution to model selection, each choice of monomial order results in a different minimal PDS, with each one yielding different hypotheses about the underlying biological network. The following example illustrates this claim.

Lactose metabolism in E.coli is controlled by the lac operon, a genetic system made up of simultaneously transcribed genes. It is said that the lac operon $(x)$ is ON (lactose is metabolized) when the activating protein CAP $(y)$ is present and when the inhibiting protein $\operatorname{lacI}(z)$ is absent. This behavior can be described by the Boolean function $f=y \wedge \neg z$; as a polynomial over the finite field $\mathbb{F}_{2}$, we can write $f=y(z+1)=y z+y$. If we consider the inputs $\mathbb{X}=\{(1,0,0),(0,1,0),(1,0,1)\}$ representing Boolean states for the lac operon, CAP, and lacI respectively, then the ideal of polynomials vanishing on $\mathbb{X}$ has two Gröbner bases, namely $\left\{x^{2}+x, z^{2}+z, y+x+1, x z+z\right\}$ and $\left\{y^{2}+y, z^{2}+z, x+y+1, y z\right\}$. The normal forms of $f$ are $x+1$ and $y$ respectively. Note that the function $f$ is selected as a model using the first Gröbner basis while a different model is selected using the second Gröbner basis.

Computing all possible minimal PDSs requires computing the Gröbner fan of the ideal which is computationally expensive, even in the finite field case. The authors in [5] posed the question of finding data sets whose corresponding ideals have a small number, possibly a unique reduced Gröbner basis, or whose Gröbner fans consist of a single cone. Their motivation was a desire to minimize the number of associated models, each with a different set of predictions.

Similar problems arise in the branch of statistics called combinatorial design of experiments (see [15] and [9], Tutorial 92, for an introduction to this topic). In the context of a field $K$, functions which fit data in $\mathbb{X} \subseteq K^{n}$ lie in the coordinate ring $K[\mathbb{X}]:=K\left[x_{1}, \ldots, x_{n}\right] / \mathcal{I}(\mathbb{X})$. Then the coset $f+\mathcal{I}(\mathbb{X})$ describes the set of models which fit the input data in $\mathbb{X}$ and one model is chosen by computing the normal form of $f \in K\left[x_{1}, \ldots, x_{n}\right]$ with respect to a Gröbner basis of the ideal of points $\mathcal{I}(\mathbb{X})$. Changing term orderings results in potentially different normal forms, i.e. different models.

The first main goal of this paper is to identify classes of ideals in polynomial rings which have a unique reduced Gröbner basis. In Section 2 we introduce fundamental tools such as G-basic sets, GFan numbers, and linear shifts (see Definitions 2.2, 2.3, and 2.6). Then it is shown in Theorem 2.7 that ideals related by a linear shift share the same number of G-basic sets, equivalently the same GFan number. Finally the classical notion of an ideal of points is recalled together with the notion of a grid of points.

Section 3 starts with Theorem 3.3 which provides a characterization of ideals whose GFan number is 1 . Such ideals turn out to have also a unique basic set as shown in Corollary 3.4. Then the important notion of a distraction is recalled. It is shown that distractions and their linear shifts provide a large class of ideals with GFan number equal to 1 (see Theorem 3.7 and Corollary 3.10 ). The last subsection of this section focuses on natural distractions and associated 
staircases. Their strong connection is highlighted in Proposition 3.19.

Section 4 contains the most relevant results of the paper. It is well-known that every zero-dimensional ideal in $P=K\left[x_{1}, \ldots, x_{n}\right]$ contains $n$ univariate polynomials, one for each indeterminate. Accordingly, we consider an ideal $J$ in $P$ generated by $n$ univariate polynomials, one for each indeterminate, and Definition 4.6 describes how two ideals $I_{1}$ and $I_{2}$ which contain $J$ can be considered to be complementary with respect to $J$. The main Theorem 4.8 shows that complementary ideals have the same Gröbner fan, hence the same GFan number, and then Corollary 4.11 provides good classes of complementary ideals. The paper is concluded in Section [5] where some applications of the theory developed before and some hints to future research are illustrated.

Basic definitions and results are taken from [8], [9], and [10], with examples computed in CoCoA-5 [1] to allow the interested reader to check the computations directly.

\section{Background}

Let $K$ be a field, $P=K\left[x_{1}, \ldots, x_{n}\right]$ a polynomial ring, and $I$ an ideal in $P$. We recall that $\mathbb{T}^{n}$ is the monoid of power products in the indeterminates $x_{1}, \ldots, x_{n}$ and that a non-empty subset $\mathcal{O}$ of $\mathbb{T}^{n}$ is called an order ideal if it is closed under division (see [9], Definition 6.4.3). If $\sigma$ is a term ordering, the set $\mathbb{T}^{n} \backslash \operatorname{LT}_{\sigma}(I)$ is denoted by $\mathcal{O}_{\sigma}(I)$. It is well-known that $\mathcal{O}_{\sigma}(I)$ is an order ideal and the residue classes of its elements form a $K$-basis of $P / I$ (see for instance [8], Corollary 2.4.11). It is also well-known that, given $I$, there are order ideals which are not of type $\mathcal{O}_{\sigma}(I)$; nevertheless the residue classes of their elements form a $K$-basis of $P / I$. The following example taken from [9] (see Example 6.4.2) is a case in point.

Example 2.1. Consider the ideal $I=\left\langle x^{2}+x y+y^{2}, x^{3}, x^{2} y, x y^{2}, y^{3}\right\rangle$ in $\mathbb{Q}[x, y]$. This ideal is symmetric with respect to switching $x$ and $y$. Since the leading term of $x^{2}+x y+y^{2}$ is either $x^{2}$ or $y^{2}$, the ideal $I$ has two possible leading term ideals, namely the ideals $J_{1}=\left\langle x^{2}, x y^{2}, y^{3}\right\rangle$ and $J_{2}=\left\langle x^{3}, x^{2} y, y^{2}\right\rangle$. Neither is symmetric. Thus they do not give rise to symmetric vector space bases of $\mathbb{Q}[x, y] / I$. However, the set of terms $\mathcal{O}=\left\{1, x, y, x^{2}, y^{2}\right\}$ is symmetric and represents a vector space basis of $\mathbb{Q}[x, y] / I$.

These considerations motivate the following definition.

Definition 2.2. An order ideal $\mathcal{O}$ such that the classes of its elements form a $K$-basis of $P / I$ is called a basic set for $I$. If there exists a term ordering $\sigma$ such that $\mathcal{O}=\mathcal{O}_{\sigma}(I)$, it is called a G-basic set for $I$. If we want to specify that a G-basic set is obtained using $\sigma$, we call it a $\sigma$-basic set.

Let $I$ be a zero-dimensional ideal with $\operatorname{dim}_{K}(P / I)=s<\infty$, let $\sigma$ be a term ordering, and let $\mathcal{O}$ be an order ideal with $s$ elements. The normal forms of

the elements in $\mathcal{O}$ with respect to $\sigma$ are linear combinations of the elements of 
$\mathcal{O}_{\sigma}(I)$, and hence can be represented by an $s \times s$ matrix, say $M$. It is then clear that $\mathcal{O}$ is a basic set for $I$ if and only if $M$ is invertible.

Some relations between basic sets and $\sigma$-basic sets are described in [4], Section 2. For zero-dimensional ideals, basic sets are the main building blocks of the theory of border bases (see [9], Section 6.4 for the introduction to that theory) which is outside the scope of the present paper.

As mentioned above, the authors in [5] and others raised the question of properties of $\mathbb{X}$ that guarantee $\mathcal{I}(\mathbb{X})$ has a unique reduced Gröbner basis, hence a unique G-basic set: such data sets have uniquely identifiable models. To count the number of G-basic sets of an ideal, we use the notion of the Gröbner fan which was introduced in 14]. It is a subdivision of the closed non-negative orthant $\mathbb{R}_{+}^{n}$ made with a finite number of polyhedral cones, such that the cones are in one-to-one correspondence to the G-basic sets for $I$.

Definition 2.3. Let $I$ be an ideal in $P$ and let GFan $(I)$ be the Gröbner fan of $I$. The number of G-basic sets for $I$, equivalently the number of leading term ideals of $I$, is called the GFan number of $I$, and is denoted by $\operatorname{GFNum}(I)$, since it coincides with the number of polyhedral cones in $\operatorname{GFan}(I)$.

We point out that the definition does not count the number of different reduced Gröbner bases, as shown with the help of the following easy examples.

Example 2.4. Let $I=\langle f\rangle \subset K[x, y]$, where $f=x+y$ and $K$ is any field. One can argue that for every term ordering $\{f\}$ is the reduced Gröbner basis. However, for every term ordering $\sigma$ with $x>y$ we have $\operatorname{LT}(I)=\langle x\rangle$ and the corresponding G-basic set is $\left\{y^{n} \mid n \in \mathbb{N}\right\}$. For every term ordering $\sigma$ with $y>x$ we have $\operatorname{LT}(I)=\langle y\rangle$ and the corresponding G-basic set is $\left\{x^{n} \mid n \in \mathbb{N}\right\}$. Consequently we have $\operatorname{GFNum}(I)=2$.

Example 2.5. Let $I=\langle x+y+z\rangle \subset K[x, y, z]$, where $K$ is any field. In this case we have $\operatorname{GFNum}(I)=3$ since the only possible leading term ideals of $I$ are $\langle x\rangle,\langle y\rangle,\langle z\rangle$.

Since a topic of this paper is to identify ideals which have the same GFan number, we note that some affine transformations do not affect leading terms. This observation motivates the following definition.

Definition 2.6. An affine transformation $\Phi: P \rightarrow P$ defined by $x_{i} \mapsto a_{i} x_{i}+b_{i}$ where $a_{i} \in K \backslash\{0\}, b_{i} \in K$ for $i=1, \ldots, n$ is called a linear shift of $P$.

Proposition 2.7. Let $\Phi$ be a linear shift of $P$ and $I$ an ideal in $P$.

(a) The ideals $I$ and $\Phi(I)$ have the same G-basic sets.

(b) We have $\operatorname{GFNum}(I)=\operatorname{GFNum}(\Phi(I))$.

Proof. To prove (a), let $\sigma$ be a term ordering on $\mathbb{T}^{n}$ and let $f$ be a non-zero polynomial in $I$. It is clear that $\operatorname{LT}_{\sigma}(f)=\mathrm{LT}_{\sigma}(\Phi(f))$ which implies the inclusion $\operatorname{LT}_{\sigma}(I) \subseteq \operatorname{LT}_{\sigma}(\Phi(I))$. But $\Phi$ is an isomorphism and its inverse is 
also a linear shift, hence we get the other inclusion. Consequently we have $\operatorname{LT}_{\sigma}(I)=\operatorname{LT}_{\sigma}(\Phi(I))$ for every term ordering $\sigma$ which implies that $I$ and $\Phi(I)$ have the same G-basic sets

Claim (b) follows from (a), thereby completing the proof.

Example 2.8. Let us return to the ideal $I$ in Example 2.1. Consider the linear shift $\Phi=(x+1, y-2)$. Then $\Phi(I)=\left\langle(x+1)^{2}+(x+1)(y-2)+(y-2)^{2},(x+\right.$ $\left.1)^{3},(x+1)^{2}(y-2),(x+1)(y-2)^{2},(y-2)^{3}\right\rangle$. Note that $\Phi(I)$ also has two leading term ideals, namely the same minimally generated ideals $J_{1}=\left\langle x^{2}, x y^{2}, y^{3}\right\rangle$ and $J_{2}=\left\langle x^{3}, x^{2} y, y^{2}\right\rangle$ as $I$ above. Indeed, $\operatorname{GFNum}(I)=\operatorname{GFNum}(\Phi(I))=2$.

In areas such as design of experiments, ideals are constructed from data as was described in the introduction. As such, we call a tuple $\left(c_{1}, \ldots, c_{n}\right) \in K^{n}$ a point, corresponding to the linear maximal ideal $\mathfrak{m}=\left\langle x_{1}-c_{1}, \ldots, x_{n}-c_{n}\right\rangle \in P$. Furthermore the vanishing ideal $\mathcal{I}(\mathbb{Y})$ of a finite set $\mathbb{Y}$ of $s$ points is a zerodimensional radical ideal in $P$ of type $\mathcal{I}(\mathbb{Y})=\mathfrak{m}_{1} \cap \cdots \cap \mathfrak{m}_{s}$, and which we also call an ideal of points. For an introduction to ideals of points, see [9], Section 6.3 ; for methods to efficiently compute them and other zero-dimensional ideals, see [2] and [3].

\section{Ideals with One Reduced Gröbner Basis}

In this section, we look for conditions which guarantee that an ideal $I$ has $\operatorname{GFNum}(I)=1$. We assume that $K$ is any a field and $P=K\left[x_{1}, \ldots, x_{n}\right]$ is a polynomial ring. Where specific conditions for $K$ are required, we will note it as necessary.

\subsection{General Results}

We start this subsection by recalling the notion of $\operatorname{Supp}(f)$ (see for instance [8] Definition 1.1.11). Let $f \in P$ and let $f=\sum_{i=1}^{r} c_{i} t_{i}$ where $c_{i} \in K$ and $t_{i} \in \mathbb{T}^{n}$. Then the support of $f$ is defined as $\operatorname{Supp}(f)=\left\{t_{i} \mid c_{i} \neq 0\right\}$. Notice that $\operatorname{Supp}(0)=\emptyset$.

Definition 3.1. A polynomial $f \in P$ is called factor-closed if there exists $t \in \operatorname{Supp}(f)$ such that all $t^{\prime} \in \operatorname{Supp}(f)$ have the property that $t^{\prime}$ divides $t$.

Lemma 3.2. Let $I \subset P$ be an ideal. Let $\sigma$ be a term ordering and $G$ be a minimal monic $\sigma$-Gröbner basis of I. Assume that every polynomial in $G$ is factor-closed.

(a) The set $G$ is the reduced $\sigma$-Gröbner basis of $I$.

(b) We have $\operatorname{GFNum}(I)=1$. 
Proof. Let us prove claim (a). For contradiction assume that $G$ is not reduced. Since it is minimal and monic, there exist $i, j \in\{1, \ldots s\}$ and a power product $\tilde{t} \in \operatorname{Supp}\left(g_{i}\right)$ such that $\operatorname{LT}_{\sigma}\left(g_{j}\right) \mid \tilde{t}$. Since $g_{i}$ is factor-closed we deduce that $\operatorname{LT}_{\sigma}\left(g_{j}\right) \mid \operatorname{LT}_{\sigma}\left(g_{i}\right)$, a contradiction to the minimality of $G$.

The proof of (b) follows from the observation that for every $i \in\{1, \ldots, s\}$ the leading term of $g_{i}$ is the same for every term ordering, hence $G$ is the reduced Gröbner basis of $I$ for every term ordering.

Theorem 3.3. Let $I \subset P$ be an ideal. The following conditions are equivalent.

(a) There exists a term ordering $\sigma$ and a minimal monic $\sigma$-Gröbner basis $G$ of I such that all the polynomials in $G$ are factor-closed.

(b) There exists a term ordering $\sigma$ such that all the polynomials in the reduced $\sigma$-Gröbner basis of I are factor-closed.

(c) We have $\operatorname{GFNum}(I)=1$.

Proof. From Lemma 3.2, we deduce that Claims (a) and (b) are equivalent and that (a) $\Rightarrow(\mathrm{c})$. Next we prove $(\mathrm{c}) \Rightarrow(\mathrm{b})$. By contradiction we assume that there exists $i$ and a power product $\tilde{t} \in \operatorname{Supp}\left(g_{i}\right)$ such that $\tilde{t}$ does not divide $\operatorname{LT}_{\sigma}\left(g_{i}\right)$. We let $t^{\prime}=\tilde{t} / \operatorname{gcd}\left(\tilde{t}, \operatorname{LT}_{\sigma}\left(g_{i}\right)\right)$ and $\left.t=\mathrm{LT}_{\sigma}\left(g_{i}\right)\right) / \operatorname{gcd}\left(\tilde{t}, \mathrm{LT}_{\sigma}\left(g_{i}\right)\right)$. Then $t^{\prime}$ and $t$ are coprime and $t^{\prime} \neq 1$. Therefore there exists $x_{j}$ such that $x_{j} \mid t^{\prime}$ and $x_{j} \nmid t$. Let $\tau$ be the lexicographic term ordering with $x_{j}>_{\tau} x_{i}$ for $i \neq j$. Then $\tilde{t}>_{\tau} \operatorname{LT}_{\sigma}\left(g_{i}\right)$ and hence the reduced $\tau$-Gröbner basis of $I$ is different from $G$. This is a contradiction and the proof is complete.

In the recent preprint [6], related results are proved for so-called neural ideals, which are generated by certain factor-closed generalizations of monomials (pseudomonomials) in Boolean rings.

Theorem 3.3 gives an efficient way to check whether an ideal $I$ has a unique reduced Gröbner basis: in fact, one can simply inspect each minimal generator for being factor-closed. This theorem also provides interesting consequences, as described in the following corollaries.

Corollary 3.4. Let $I$ be an ideal in $P$ with $\operatorname{GFNum}(I)=1$, and let $\mathcal{O}(I)$ be the unique $G$-basic set for $I$. Then $\mathcal{O}(I)$ is also the unique basic set for $I$.

Proof. Let $G=\left\{g_{1}, \ldots, g_{s}\right\}$ be the unique reduced Gröbner basis of $I$. For contradiction, assume that there exists a basic set $\mathcal{O}$ for $I$ such that $\mathcal{O} \neq \mathcal{O}(I)$, and let $t \in \mathcal{O} \backslash \mathcal{O}(I)$. By definition of Gröbner basis, there exists $i$ such that $\mathrm{LT}\left(g_{i}\right) \mid t$. From the theorem we know that $g_{i}$ is factor-closed, hence every power product in $\operatorname{Supp}\left(g_{i}\right)$ divides $t$. On the other hand $\mathcal{O}$ is an order ideal, hence every power product in $\operatorname{Supp}\left(g_{i}\right)$ is in $\mathcal{O}$. Therefore we get a non-trivial linear combination of elements of $\mathcal{O}$ which is zero in $P / I$, thus a contradiction.

Corollary 3.5. Let $\Phi$ be a linear shift of $P$. Let $I$ be a monomial ideal in $P$, and $\mathcal{O}(I)$ the set of power products which are not divisible by any power product in $I$. 
(a) We have $\operatorname{GFNum}(I)=1$ and $\mathcal{O}(I)$ is the unique basic set for $I$.

(b) We have $\operatorname{GFNum}(\Phi(I))=1$ and $\mathcal{O}(I)$ is the unique basic set for $\Phi(I)$.

Proof. To prove Claim (a), we observe that $\mathcal{O}(I)$ is the unique G-basic set for $I$ by Theorem 3.3. Then the conclusion follows from Corollary 3.4.

Claim (b) follows from (a) and Theorem 2.7

When $I$ has a unique reduced Gröbner basis, the above results show that linear shifts preserve leading terms as well as basic sets. In the remainder of this section and in Section 4, we will see similar results for other types of ideals.

We observe that a linear shift is composed of two types of shifts, namely $\Phi_{1}$ of type $x_{i} \mapsto a_{i} x_{i}$ and $\Phi_{2}$ of type $x_{i} \mapsto x_{i}+b_{i}$. Clearly, if $I$ is a monomial ideal we have $I=\Phi_{1}(I)$, so the only non-trivial part of Corollary 3.5 is that $\operatorname{GFNum}\left(\Phi_{2}(I)\right)=1$.

\subsection{Distractions}

In Corollary 3.5 we have seen a modification of monomial ideals which produces ideals with GFan number equal to 1 . In the literature there is another interesting construction which yields the same result. For a complete introduction to the theory of distractions, see [9].

Definition 3.6. Let $K$ be an infinite field. For $i=1, \ldots, n$, let $\pi_{i}=\left(c_{i 1}, c_{i 2}, \ldots\right)$ be a sequence with $c_{i j} \in K$ and $c_{i j} \neq c_{i k}$ for every $j \neq k$. Set $\pi=\left(\pi_{1}, \ldots, \pi_{n}\right)$.

1. For every power product $t=x_{1}^{\alpha_{1}} \cdots x_{n}^{\alpha_{n}}$ in $\mathbb{T}^{n}$, the polynomial

$$
D_{\pi}(t)=\prod_{i=1}^{\alpha_{1}}\left(x_{1}-c_{1 i}\right) \cdot \prod_{i=1}^{\alpha_{2}}\left(x_{2}-c_{2 i}\right) \cdots \prod_{i=1}^{\alpha_{n}}\left(x_{n}-c_{n i}\right)
$$

is called the distraction of $t$ with respect to $\pi$.

2. Let $I$ be a monomial ideal in $P$, and let $\left\{t_{1}, \ldots, t_{s}\right\}$ be the unique minimal monomial system of generators of $I$. Then we say that the ideal $D_{\pi}(I)=\left\langle D_{\pi}\left(t_{1}\right), \ldots, D_{\pi}\left(t_{s}\right)\right\rangle$ is the distraction of $I$ with respect to $\pi$.

Theorem $3.7(9])$. Let I be a monomial ideal in $P$, let $\left\{t_{1}, \ldots, t_{s}\right\}$ be a minimal set of power products which generates $I$, let $\pi=\left(\pi_{1}, \ldots, \pi_{n}\right)$ be sequences of pairwise distinct elements in $K$, and let $D_{\pi}(I)=\left\langle D_{\pi}\left(t_{1}\right), \ldots, D_{\pi}\left(t_{s}\right)\right\rangle$ be the corresponding distraction of $I$.

(a) The ideal $D_{\pi}(I)$ is radical.

(b) The set $\left\{D_{\pi}\left(t_{1}\right), \ldots, D_{\pi}\left(t_{s}\right)\right\}$ is the reduced $\sigma$-Gröbner basis of $D_{\pi}(I)$ for every term ordering $\sigma$.

(c) We have $\operatorname{GFNum}\left(D_{\pi}(I)\right)=1$.

See Theorem 6.2.12 in [9] for a proof. 
Example 3.8. Let $K=\mathbb{Q}$. Consider $I=\left\langle t_{1}, t_{2}\right\rangle$, where $t_{1}=x^{3} y$ and $t_{2}=$ $x^{2} y^{4}$. Set $\pi_{1}=(3,2,5), \pi_{2}=(2,-1,3,12)$. As the elements in $\pi_{1}, \pi_{2}$ are pairwise distinct, we can make the distraction of $I$ with respect to $\pi=\left(\pi_{1}, \pi_{2}\right)$ : $D_{\pi}(I)=\left\langle D_{\pi}\left(t_{1}\right), D_{\pi}\left(t_{2}\right)\right\rangle$ where

$$
\begin{gathered}
D_{\pi}\left(t_{1}\right)=(x-3)(x-2)(x-5)(y-2), \\
D_{\pi}\left(t_{2}\right)=(x-3)(x-2)(y-2)(y+1)(y-3)(y-12) .
\end{gathered}
$$

According to Theorem 3.7, the ideal $D_{\pi}(I)$ is radical and $\left\{D_{\pi}\left(t_{1}\right), D_{\pi}\left(t_{2}\right)\right\}$ is the reduced $\sigma$-Gröbner basis for every $\sigma$, and so $\operatorname{GFNum}\left(D_{\pi}(I)\right)=1$.

The assumption that $K$ is infinite guarantees the existence of a distraction of all monomial ideals. However, in order to define the distraction $D_{\pi}(I)$ of a single monomial ideal $I$, it suffices to specify the first $d_{i}$ elements of the sequence $\pi_{i}$ where $d_{i}=\max \left\{\operatorname{deg}_{x_{i}}\left(t_{j}\right) \mid j \in\{1, \ldots, s\}\right\}$ for $i=1, \ldots, n$. In particular, to distract a monomial ideal $I$, it is sufficient to use finite tuples of elements. Consequently we do not have to assume that $K$ is infinite, as long as $K$ has sufficiently many elements.

Example 3.9. Consider the ideal in Example 3.8. As the largest exponent is 4, we need a field which has at least four elements. So $\mathbb{F}_{2}$ and $\mathbb{F}_{3}$ are excluded. On the other hand, if $K=\mathbb{F}_{5}$ we can choose $\pi=\left(\pi_{1}, \pi_{2}\right)$ where $\pi_{1}=(1,3,0)$, $\pi_{2}=(0,1,2,3)$. Then we get $D_{\pi}\left(t_{1}\right)=(x-1)(x-3) x y, D_{\pi}\left(t_{2}\right)=(x-1)(x-$ 3) $y(y-1)(y-2)(y-3)$.

As a consequence of Theorem 3.7 and Proposition 2.7, we get the following result.

Corollary 3.10. We make the same assumptions as in Theorem 3.7 with the extra-condition that $I$ is a zero-dimensional ideal.

(a) The ideal $D_{\pi}(I)$ is an ideal of points and $\operatorname{GFNum}\left(D_{\pi}(I)\right)=1$.

(b) If $\Phi$ is a linear shift of $P$, the ideal $\Phi\left(D_{\pi}(I)\right)$ is an ideal of points, and $\operatorname{GFNum}\left(\Phi\left(D_{\pi}(I)\right)\right)=1$.

Proof. Claim (a) follows from [9], Theorem 6.2.12(a) and Theorem 3.7(c).

Claim (b) follows from (a) and Proposition 2.7.

The following examples illustrate interesting outcomes of this corollary.

Example 3.11. Consider the monomial ideal $J=\left\langle x^{4}, y^{3}, x^{2} y, x y^{2}\right\rangle \subset \mathbb{Q}[x, y]$. We will show how to construct a set of points $\mathbb{X}$ such that its ideal of points $\mathcal{I}(\mathbb{X})$ is a distraction of $J$.

Since the first two generators of $J$ are not mixed and have degrees larger than the powers of $x$ and $y$ in the other two generators, we can use $x^{4}$ and $y^{3}$ to construct two sequences and two polynomials. Let $\pi_{1}$ be any sequence with at least 4 entries, say $\left(0, \frac{1}{5}, 2,-1, \ldots\right)$. 
Then the polynomial $f_{1}:=x\left(x-\frac{1}{5}\right)(x-2)(x+1)$ is the distraction of $x^{4}$ with respect to $\pi_{1}$. Similarly let $\pi_{2}$ be any sequence with at least 3 entries, say $(0,1,2, \ldots)$. Then $f_{2}:=y(y-1)(y-2)=D_{\pi_{2}}\left(y^{3}\right)$. Set $\pi=\left(\pi_{1}, \pi_{2}\right)$. Now we can construct the distractions of the other two power products, namely $f_{3}:=x\left(x-\frac{1}{5}\right) y$ and $f_{4}:=x y(y-1)$.

Consider the ideal $I=\left\langle f_{1}, f_{2}, f_{3}, f_{4}\right\rangle$. Notice that $I$ is the distraction of $J$ with respect to $\pi$. Furthermore, $I$ is the ideal of the points

$$
\mathbb{X}=\left\{(0,0),(0,1),(0,2),\left(\frac{1}{5}, 0\right),\left(\frac{1}{5}, 1\right),(2,0),(-1,0)\right\} .
$$

We observe that $\mathcal{I}(\mathbb{X})=D_{\pi}(J)$ and $\operatorname{GFNum}\left(D_{\pi}(J)\right)=1$ follows from Corollary 3.10 (a).

The following examples show that if we do not follow the rigid order in the choice of the constants imposed by the definition of distraction, unexpected things can happen.

Example 3.12. If we consider the polynomials $f_{1}, f_{2}$ of Example 3.11 and the two polynomials $\ell_{3}=(x-2)(y-1)(y-2), \quad \ell_{4}=(x+1)\left(x-\frac{1}{5}\right)(y-1)$, then the ideal $I_{4}=\left\langle f_{1}, f_{2}, \ell_{3}, \ell_{4}\right\rangle$ is not a distraction of $J$ for any permutation of the tuples $\left(0, \frac{1}{5}, 2,-1\right)$ and $(0,1,2)$. However, it has the unique reduced Gröbner basis $G=\left\{x^{4}-\frac{6}{5} x^{3}-\frac{9}{5} x^{2}+\frac{2}{5} x, y^{2}-3 y+2, x^{2} y-x^{2}+\frac{4}{5} x y-\frac{4}{5} x-\frac{1}{5} y+\frac{1}{5}\right\}$.

From the equalities

$$
\begin{array}{ll}
x^{4}-\frac{6}{5} x^{3}-\frac{9}{5} x^{2}+\frac{2}{5} x & =(x+1)\left(x-\frac{1}{5}\right)(x)(x-2) \\
y^{2}-3 y+2 & =(y-1)(y-2) \\
x^{2} y-x^{2}+\frac{4}{5} x y-\frac{4}{5} x-\frac{1}{5} y+\frac{1}{5} & =(y-1)(x+1)\left(x-\frac{1}{5}\right) .
\end{array}
$$

we see that $I_{4}$ is the distraction of the monomial ideal $\left\langle x^{4}, y^{2}, x^{2} y\right\rangle$ with respect to $\pi=\left(\pi_{1}, \pi_{2}\right)$ where $\pi_{1}=\left(-1, \frac{1}{5}, 0,2\right)$ and $\pi_{2}=(1,2)$.

Example 3.13. Consider the ideal $I$ of the following set of four points

$$
\{(0,0,0),(1,0,0),(1,1,0),(1,1,1)\} .
$$

Its reduced Gröbner basis with respect to $\sigma=$ DegRevLex is

$$
\left\{z^{2}-z, y z-z, x z-z, y^{2}-y, x y-y, x^{2}-x\right\} .
$$

All polynomials in this basis are factor-closed, hence we have $\operatorname{GFNum}(I)=1$ by Theorem 3.3. We have $\operatorname{LT}_{\sigma}(I)=\left\langle z^{2}, y z, x z, y^{2}, x y, x^{2}\right\rangle$. However, $\operatorname{LT}_{\sigma}(I)$ is not a distraction of $I$ since we have the equalities

$$
y z-z=z(\mathbf{y}-\mathbf{1}) \text { and } \quad x y-y=(x-1) \mathbf{y}
$$




\subsection{Natural Distractions and Staircases}

In this subsection we introduce an interesting family of distractions. We recall that an ideal is called irreducible if it cannot be written as the intersection of two ideals, both of which properly contain it, and use some results from [9].

Proposition 3.14. Let $K$ be a field and let $P=K\left[x_{1}, \ldots, x_{n}\right]$.

(a) Every proper ideal in $P$ is a finite intersection of irreducible ideals.

(b) A monomial ideal $I$ in $P$ is irreducible if and only if it is of the form $I=\left\langle x_{i_{1}}^{d_{1}}, \ldots, x_{i_{s}}^{d_{s}}\right\rangle$ with $1 \leq i_{1}<\cdots<i_{s} \leq n$ and $d_{1}, \ldots, d_{s} \in \mathbb{N}$.

(c) A zero-dimensional monomial ideal is irreducible if and only if it is of the form $I=\left\langle x_{1}^{d_{1}}, \ldots, x_{n}^{d_{n}}\right\rangle$ with $d_{1}, \ldots, d_{s} \in \mathbb{N}$.

Proof. For Claim (a) see 9], Proposition 5.6.17. For Claim (b) see 9], Proposition 6.2.11. Claim (c) follows immediately from (b).

From Corollary 3.5 we know that associated to every monomial ideal there is a unique basic set $\mathcal{O}(I)$, the set of power products which are not divisible by any power product in $I$. This observation motivates the following definition. Since the exponents of every monomial are natural numbers, they can be viewed as elements of any field $K$ via the natural map $\mathbb{N} \rightarrow K$.

Definition 3.15. Let $K$ be a field and let $P=K\left[x_{1}, \ldots, x_{n}\right]$.

1. Given $t=x_{1}^{a_{1}} \cdots x_{n}^{a_{n}}$, we say that $p(t):=\left(a_{1}, \ldots, a_{n}\right) \in K^{n}$ is the point associated to $t$.

2. Let $I$ be a monomial ideal and let $\mathcal{O}(I)$ be the unique basic set associated to $I$. Then the set $\{p(t) \mid t \in \mathcal{O}(I)\}$ is called the staircase of points associated to $I$ and denoted by $\operatorname{Stair}(I)$.

Example 3.16. Let $I=\left\langle x^{2}, x y z^{2}, y^{2}, z^{3}\right\rangle \subset \mathbb{Q}[x, y, z]$. Then we have

$$
\mathcal{O}(I)=\left\{1, z, z^{2}, y, y z, y z^{2}, x, x z, x z^{2}, x y, x y z\right\} .
$$

Hence we get

$$
\begin{array}{r}
\operatorname{Stair}(I)=\{(0,0,0),(0,0,1),(0,0,2),(0,1,0),(0,1,1)(0,1,2), \\
(1,0,0),(1,0,1),(1,0,2),(1,1,0),(1,1,1)\} .
\end{array}
$$

Lemma 3.17. Assume $I_{1}, I_{2}$ are zero-dimensional monomial ideals in $P$. Let $\pi_{1}, \ldots, \pi_{n}$ be sequences of pairwise distinct elements of the field $K$, and set $\pi=\left(\pi_{1}, \ldots, \pi_{n}\right)$.

(a) $D_{\pi}\left(I_{1} \cap I_{2}\right)=D_{\pi}\left(I_{1}\right) \cap D_{\pi}\left(I_{2}\right)$.

(b) $\mathcal{O}\left(I_{1}+I_{2}\right)=\mathcal{O}\left(I_{1}\right) \cap \mathcal{O}\left(I_{2}\right)$

(c) $\mathcal{O}\left(I_{1} \cap I_{2}\right)=\mathcal{O}\left(I_{1}\right) \cup \mathcal{O}\left(I_{2}\right)$. 
(d) $\operatorname{Stair}\left(I_{1} \cap I_{2}\right)=\operatorname{Stair}\left(I_{1}\right) \cup \operatorname{Stair}\left(I_{2}\right)$.

Proof. Claim (a) follows from [9], Proposition 6.2.10.

To prove Claim (b), notice that the inclusions $I_{1} \subseteq I_{1}+I_{2}$ and $I_{2} \subseteq I_{1}+I_{2}$ imply $\mathcal{O}\left(I_{1}+I_{2}\right) \subseteq \mathcal{O}\left(I_{1}\right)$ and $\mathcal{O}\left(I_{1}+I_{2}\right) \subseteq \mathcal{O}\left(I_{2}\right)$. It follows that $\mathcal{O}\left(I_{1}+I_{2}\right) \subseteq$ $\mathcal{O}\left(I_{1}\right) \cap \mathcal{O}\left(I_{2}\right)$. On the other hand, if $t$ is a power product with $t \notin I_{1}$ and $t \notin I_{2}$, then $t \notin I_{1}+I_{2}$ and the claim is proved.

Let us now prove (c). From the inclusions $I_{1} \cap I_{2} \subseteq I_{1}$ and $I_{1} \cap I_{2} \subseteq I_{2}$ we get the inclusions $\mathcal{O}\left(I_{1}\right) \subseteq \mathcal{O}\left(I_{1} \cap I_{2}\right)$ and $\mathcal{O}\left(I_{2}\right) \subseteq \mathcal{O}\left(I_{1} \cap I_{2}\right)$, hence the inclusion $\mathcal{O}\left(I_{1}\right) \cup \mathcal{O}\left(I_{2}\right) \subseteq \mathcal{O}\left(I_{1} \cap I_{2}\right)$. To conclude the proof, we need to show that the two sets have the same number of elements. On the other hand, if $I$ is a zero-dimensional monomial ideal, the number of elements of $\mathcal{O}(I)$ is finite. Since we have

$$
\operatorname{card}\left(\mathcal{O}\left(I_{1}\right) \cup \mathcal{O}\left(I_{2}\right)\right)=\operatorname{card}\left(\mathcal{O}\left(I_{1}\right)\right)+\operatorname{card}\left(\mathcal{O}\left(I_{2}\right)\right)-\operatorname{card}\left(\mathcal{O}\left(I_{1}\right) \cap \mathcal{O}\left(I_{2}\right)\right)
$$

we need to prove the equality

$$
\operatorname{card}\left(\mathcal{O}\left(I_{1} \cap I_{2}\right)\right)=\operatorname{card}\left(\mathcal{O}\left(I_{1}\right)\right)+\operatorname{card}\left(\mathcal{O}\left(I_{2}\right)\right)-\operatorname{card}\left(\mathcal{O}\left(I_{1}\right) \cap \mathcal{O}\left(I_{2}\right)\right) .
$$

To show this equality, we construct the exact sequence of $K$-vector spaces

$$
0 \rightarrow P /\left(I_{1} \cap I_{2}\right) \rightarrow\left(P / I_{1}\right) \oplus\left(P / I_{2}\right) \rightarrow P /\left(I_{1}+I_{2}\right) \rightarrow 0
$$

defined by the map

$$
P /(I \cap J) \rightarrow(P / I) \oplus(P / J) \text { given by } f+(I \cap J) \mapsto(f+I, f+J)
$$

and the map

$$
(P / I) \oplus(P / J) \rightarrow P /(I+J) \text { given by }(f+I, g+J) \mapsto f-g+I+J .
$$

From the exact sequence, we get the equality

$$
\operatorname{card}\left(\mathcal{O}\left(I_{1} \cap I_{2}\right)\right)=\operatorname{card}\left(\mathcal{O}\left(I_{1}\right)\right)+\operatorname{card}\left(\mathcal{O}\left(I_{2}\right)\right)-\operatorname{card}\left(\mathcal{O}\left(I_{1}+I_{2}\right)\right) .
$$

From Claim (b) we deduce that this equality coincides with (1).

Claim (d) follows immediately from (c) and the definition of a staircase. Hence the proof is complete.

We are ready to introduce a special type of distractions.

Definition 3.18. Consider a monomial ideal $I$ in $P$. Let $d_{1}, \ldots, d_{n}$ be the maximal exponents of $x_{1}, \ldots, x_{n}$ in the minimal set of generators of $I$ and take $d=\max \left\{d_{i}\right\}$. Assume that $0,1, \ldots, d-1$ are distinct elements of $K$. Furthermore, let $\pi_{d_{i}}=\left(0,1,2,3, \ldots, d_{i}-1\right)$ and let $\pi_{\text {nat }}=\left(\pi_{d_{1}}, \ldots, \pi_{d_{n}}\right)$. Then the distraction $D_{\pi_{\text {nat }}}(I)$ is called the natural distraction (or classic distraction) of the ideal $I$.

The following proposition shows the connections between natural distractions and staircases. 
Proposition 3.19. Consider a monomial ideal $I$ in $P$. Then we have the equality $\mathcal{I}(\operatorname{Stair}(I))=D_{\pi_{\text {nat }}}(I)$.

Proof. As a first step, we prove the claim with the extra assumption that $I$ is irreducible, hence of type $I=\left\langle x_{1}^{a_{1}}, \ldots, x_{n}^{a_{n}}\right\rangle$. In this case it is easy to see that $\operatorname{Stair}(I)=\left\{\left(c_{1}, \ldots, c_{n}\right) \mid 0 \leq c_{k}<a_{k}\right.$ for $\left.k=1, \ldots, n\right\}$. Consequently we have

$$
\begin{aligned}
\mathcal{I}(\operatorname{Stair}(I)) & =\bigcap_{0 \leq c_{k}<a_{k}}\left\langle x_{1}-c_{1}, \ldots, x_{n}-c_{n}\right\rangle \\
& =\left\langle\prod_{c_{1}=1}^{a_{1}-1}\left(x_{1}-c_{1}\right), \ldots, \prod_{c_{n}=1}^{a_{n}-1}\left(x_{n}-c_{n}\right)\right\rangle \\
& =D_{\pi_{\text {nat }}}(I) .
\end{aligned}
$$

Next we prove the general claim. From Proposition 3.14 we get an equality $I=\bigcap_{k=1}^{s} J_{k}$ with $J_{k}$ irreducible for $k=1, \ldots, s$. We deduce the following equalities

$$
\begin{gathered}
\mathcal{I}(\operatorname{Stair}(I))=\mathcal{I}\left(\operatorname{Stair}\left(\cap_{k=1}^{s}\left(J_{k}\right)\right)\right) \stackrel{(1)}{=} \mathcal{I}\left(\cup_{k=1}^{s} \operatorname{Stair}\left(J_{k}\right)\right)= \\
\bigcap_{k=1}^{s} \mathcal{I}\left(\operatorname{Stair}\left(J_{k}\right)\right) \stackrel{(2)}{=} \bigcap_{k=1}^{s} D_{\pi_{\text {nat }}}\left(J_{k}\right) \stackrel{(3)}{=} D_{\pi_{\text {nat }}}\left(\cap_{k=1}^{s} J_{k}\right)=D_{\pi_{\text {nat }}}(I)
\end{gathered}
$$

where Equality (1) follows from Lemma 3.17(d), Equality (2) follows from the special case discussed above, and Equality (3) follows from Lemma 3.17(a).

The following example illustrates the special feature of natural distractions proved in the theorem above.

Example 3.20. Let $P=\mathbb{Q}[x, y]$ and let $I=\left\langle x^{5}, x^{4} y, x y^{2}, y^{4}\right\rangle$. Then we have

$$
\begin{array}{r}
D_{\pi_{\text {nat }}}(I)=\langle x(x-1)(x-2)(x-3)(x-4), x(x-1)(x-2)(x-3) y, \\
x y(y-1), y(y-1)(y-2)(y-3)\rangle .
\end{array}
$$

If we draw a picture of the power products involved, we get

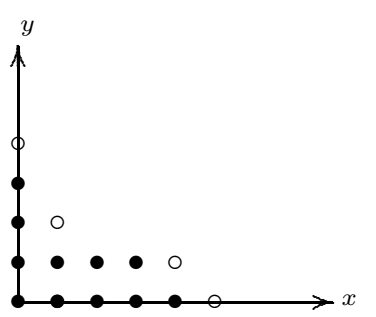

where the white dots represent the generators of $I$ and the black dots represents the power products in $\mathcal{O}(I)$. According to Proposition 3.19, we can check that the set of points defined by the ideal $D_{\pi_{\text {nat }}}(I)$ is exactly the staircase represented by the black dots. 


\section{Complementary ideals}

In this section we concentrate on zero-dimensional ideals and introduce the notion of complementary ideals (see Definition 4.6). Throughout the section, we let $K$ be a field and $P=K\left[x_{1}, \ldots, x_{n}\right]$ as before.

\subsection{Grids}

It is well-known that under the assumption that $I$ is a zero-dimensional ideal, we have $\operatorname{dim}_{K}(P / I)<\infty$ (see for instance [8], Proposition 3.7.1). Consequently, for every $i=1, \ldots, n$, the natural embedding $K\left[x_{i}\right] /\left(I \cap K\left[x_{i}\right]\right) \hookrightarrow P / I$ shows that $I \cap K\left[x_{i}\right] \neq\langle 0\rangle$, and hence $I \cap K\left[x_{i}\right]$ is a principal non-zero ideal. We denote its monic generator by $f_{I}\left(x_{i}\right)$. These facts motivate the following definition.

Definition 4.1. Let $I$ be a zero-dimensional ideal in $P$ and for $i=1, \ldots, n$, let $f_{I}\left(x_{i}\right)$ be the monic generator of $I \cap K\left[x_{i}\right]$.

1. The ideal $\left\langle f_{I}\left(x_{1}\right), \ldots, f_{I}\left(x_{n}\right)\right\rangle \subseteq I$ is called the maximal grid ideal contained in $I$ and denoted by $\operatorname{mgrid}(I)$.

2. More generally, every ideal in $P$ of type $\left\langle g_{1}\left(x_{1}\right), \ldots, g_{n}\left(x_{n}\right)\right\rangle$ and such that $\operatorname{deg}\left(g_{i}\left(x_{i}\right)\right)>0$ for every $i=1, \ldots, n$ is called a grid ideal.

When $I$ is a zero-dimensional ideal and $J=\left\langle g_{1}\left(x_{1}\right), \ldots, g_{n}\left(x_{n}\right)\right\rangle$ is a grid ideal such that $J \subseteq I$, it is clear $g_{i}\left(x_{i}\right)$ is a multiple of $f_{I}\left(x_{i}\right)$ for every $i=$ $1, \ldots, n$. This observation validates the use of "maximal" in the above definition.

Special cases of grid ideals are obtained as follows, using language from combinatorial experimental design.

Definition 4.2. Let $d_{1}, \ldots, d_{n} \in \mathbb{N}_{+}$and for $i=1, \ldots, n$, let $\left(c_{i, 1}, \ldots, c_{i, d_{i}}\right)$ be a $d_{i}$-tuple of pairwise distinct elements of $K$. Then the following set of points $\mathbb{X}=\left\{\left(c_{1, k_{1}}, \ldots, c_{n, k_{n}}\right) \quad \mid \quad 1 \leq k_{1} \leq d_{1}, \ldots, 1 \leq k_{n} \leq d_{n}\right\}$ is called a grid of points or a full design in $K^{n}$. It consists of $\prod_{i=1}^{n} d_{i}$ points. The vanishing ideal of $\mathbb{X}$ is a grid ideal generated by $\left\{g_{1}\left(x_{1}\right), \ldots, g_{n}\left(x_{n}\right)\right\}$ where $g_{i}\left(x_{i}\right)=\prod_{j=1}^{d_{i}}\left(x_{i}-c_{i j}\right)$.

Remark 4.3. Let $\mathbb{Y}$ be a set of points. The maximal grid ideal contained in $\mathcal{I}(\mathbb{Y})$ is the vanishing ideal of a set $\mathbb{X}$ of points. In agreement with Definition 4.1 the set $\mathbb{X}$ is called the minimal grid of points containing $\mathbb{Y}$.

Example 4.4. As observed in Remark 4.3, given a set of points $\mathbb{Y} \subseteq K^{n}$, its vanishing ideal $\mathcal{I}(\mathbb{Y})$ contains $n$ univariate polynomials $f_{i}\left(x_{i}\right)$ which are products of linear polynomials of type $x_{i}-c_{i j}$, and define the minimal grid $\mathbb{K}$ containing $\mathbb{Y}$. If $d_{i}=\operatorname{deg}\left(f_{i}\left(x_{i}\right)\right)$, then $f_{i}\left(x_{i}\right)$ is the distraction of $x_{i}^{d_{i}}$ with respect to any permutation of the tuple of the $c_{i j}$ 's.

We recall that for a zero-dimensional local ring $R$ with maximal ideal $\mathfrak{m}$, the socle of $R$ is defined as $\operatorname{soc}(R)=\operatorname{Ann}_{R}(\mathfrak{m})$, the annihilator of $\mathfrak{m}$. The following easy lemma collects some properties of grid ideals. 
Lemma 4.5. Let $J=\left\langle g_{1}\left(x_{1}\right), \ldots, g_{n}\left(x_{n}\right)\right\rangle$ be a grid ideal with $d_{i}=\operatorname{deg}\left(g_{i}\left(x_{i}\right)\right)$ for $i=1, \ldots, n$.

(a) The ideal $J$ is zero-dimensional.

(b) The set $\left\{g_{1}\left(x_{1}\right), \ldots, g_{n}\left(x_{n}\right)\right\}$ is the reduced $\sigma$-Gröbner basis of $J$ for every term ordering $\sigma$, and hence $\operatorname{GFNum}(J)=1$.

(c) For every term ordering $\sigma$ the residue class of $x_{1}^{d_{1}-1} x_{2}^{d_{2}-1} \cdots x_{n}^{d_{n}-1}$ generates $\operatorname{soc}\left(P / \operatorname{LT}_{\sigma}(J)\right)$.

(d) We have $\mathbb{T}^{n} \backslash \operatorname{LT}_{\sigma}(J)=\left\{t \in \mathbb{T}^{n} \mid t\right.$ divides $\left.x_{1}^{d_{1}-1} x_{2}^{d_{2}-1} \cdots x_{n}^{d_{n}-1}\right\}$ for every term ordering $\sigma$ on $\mathbb{T}^{n}$.

Proof. Claim (a) follows from the Finiteness Criterion (see [8], Proposition 3.7.1). Claim (b) follows from the fact that $\operatorname{LT}_{\sigma}\left(g_{i}\right)=x_{i}^{d_{i}}$, hence they are pairwise coprime. The other claims follow immediately.

This lemma suggests that $x_{1}^{d_{1}-1} x_{2}^{d_{2}-1} \cdots x_{n}^{d_{n}-1}$ will be denoted by $t_{s o c}(J)$. From Lemma 4.5(b) we know that if $J$ is a grid ideal we have $\operatorname{GFNum}(J)=1$. Therefore the set $\mathcal{O}_{\sigma}(J)$ is the same for every $\sigma$ and hence it will be denoted by $\mathcal{O}(J)$.

Definition 4.6. Let $J$ be a grid ideal and let $J=\mathfrak{q}_{1} \cap \cdots \cap \mathfrak{q}_{s}$ be its primary decomposition. For $0<t<s$, set $I_{1}=\mathfrak{q}_{1} \cap \cdots \cap \mathfrak{q}_{t}$, and $I_{2}=\mathfrak{q}_{t+1} \cap \cdots \cap \mathfrak{q}_{s}$. Then we say that $I_{1}, I_{2}$ are complementary ideals with respect to $J$, or simply complementary ideals, if $J$ is clear from the context.

The following lemma collects some properties of complementary ideals.

Lemma 4.7. Let $J$ be a grid ideal in $P$ and let $I_{1}, I_{2}$ be complementary ideals with respect to $J$.

(a) $J=I_{1} \cap I_{2}, \quad I_{1}+I_{2}=\langle 1\rangle, \quad I_{2}=J: I_{1}$, and $I_{1}=J: I_{2}$.

(b) There is an isomorphism of $K$-algebras $\varphi: P / I \cong P / I_{1} \times P / I_{2}$.

(c) $\operatorname{dim}_{K}(P / J)=\operatorname{dim}_{K}\left(P / I_{1}\right)+\operatorname{dim}_{K}\left(P / I_{2}\right)$, and hence we have $\operatorname{card}(\mathcal{O}(J))=\operatorname{card}\left(\mathcal{O}_{\sigma}\left(I_{1}\right)\right)+\operatorname{card}\left(\mathcal{O}_{\sigma}\left(I_{2}\right)\right)$ for every term ordering $\sigma$.

(d) If $I$ is a zero-dimensional ideal in $P$, then Claims (a), (b), and (c) hold for $J=\operatorname{mgrid}(I), I_{1}=I$, and $I_{2}=\operatorname{mgrid}(I): I$.

Proof. Claim (a) can be proved using standard facts in commutative algebra. Claim (b) follows from (a) and the Chinese Remainder Theorem (see for instance [8], Lemma 3.7.4). Claim (c) follows from (b) since the residue classes of the elements of $B_{\sigma}(I)$ form a $K$-basis of $P / I$ for any term ordering $\sigma$ and any ideal $I$ in $P$. Finally, Claim (d) is a consequence of the fact that $\operatorname{mgrid}(I)$ is a grid ideal which contains $I$.

The following result is one of the main contributions of this paper. 
Theorem 4.8. Let $J$ be a grid ideal in $P$, let $I_{1}, I_{2}$ be complementary ideals with respect to $J$, and let $\sigma$ be a term ordering.

(a) For $i=1,2$ we have $\mathcal{O}(J) \cap \operatorname{LT}_{\sigma}\left(I_{i}\right)=\mathcal{O}(J) \backslash \mathcal{O}_{\sigma}\left(I_{i}\right)$.

(b) Let $\alpha_{\sigma}: \mathcal{O}(J) \cap \operatorname{LT}_{\sigma}\left(I_{i}\right) \rightarrow \mathbb{T}^{n}$ be the map which sends $t \mapsto t_{\text {soc }}(J) / t$. Then $\alpha_{\sigma}$ is injective and induces a map $\vartheta_{\sigma}: \mathcal{O}(J) \backslash \mathcal{O}_{\sigma}\left(I_{1}\right) \rightarrow \mathcal{O}_{\sigma}\left(I_{2}\right)$ which is bijective.

(c) We have $\operatorname{GFan}\left(I_{1}\right)=\operatorname{GFan}\left(I_{2}\right)$ and hence $\operatorname{GFNum}\left(I_{1}\right)=\operatorname{GFNum}\left(I_{2}\right)$.

Proof. First, we prove Claim (a). From $I_{i} \supseteq J$, we deduce that $\operatorname{LT}\left(I_{i}\right) \supseteq \operatorname{LT}(J)$, hence $\mathcal{O}_{\sigma}\left(I_{i}\right) \subseteq \mathcal{O}(J)$ which implies the claim.

Next we prove Claim (b). The fact that $\alpha$ is injective is by construction. By contradiction, assume that $t_{\text {soc }}(J) / t \notin \mathcal{O}_{\sigma}\left(I_{2}\right)$. Then $t_{\text {soc }}(J) / t \in \mathrm{LT}_{\sigma}\left(I_{2}\right)$. We have $t_{\text {soc }}(J)=t \cdot t_{\text {soc }}(J) / t \in \mathrm{LT}_{\sigma}\left(I_{1}\right) \cdot \mathrm{LT}_{\sigma}\left(I_{2}\right) \subseteq \operatorname{LT}_{\sigma}\left(I_{1} \cdot I_{2}\right)$. Clearly $I_{1} \cdot I_{2} \subseteq J$, and so we get $t_{\text {soc }}(J) \in \mathrm{LT}_{\sigma}(I)$ which yields a contradiction by Lemma 4.5(b). Consequently we get an injective map $\mathcal{O}(J) \cap \mathrm{LT}_{\sigma}\left(I_{1}\right) \rightarrow \mathcal{O}_{\sigma}\left(I_{2}\right)$, and from (a) we can rewrite it as a map $\vartheta: \mathcal{O}(J) \backslash \mathcal{O}_{\sigma}\left(I_{1}\right) \rightarrow \mathcal{O}_{\sigma}\left(I_{2}\right)$ which is injective. Lemma 4.7(c) shows that the two sets have the same cardinality, hence we conclude that $\vartheta$ is bijective.

We observe that the set $\left.\mathcal{O}_{\sigma}\left(I_{1}\right)\right)$ is the same for every $\sigma$ which corresponds to a point inside a polyhedral cone of $\operatorname{GFan}\left(I_{1}\right)$. Therefore also the set $\left.\mathcal{O}(J) \backslash \mathcal{O}_{\sigma}\left(I_{1}\right)\right)$ is the same, hence we deduce from Claim (b) that also the set $\left.\mathcal{O}_{\sigma}\left(I_{2}\right)\right)$ is the same, and the proof is complete.

Remark 4.9. In the proof of Claim (b), we used the fact that $I_{1} \cdot I_{2} \subseteq J$. Actually we have $I_{1} \cdot I_{2}=J$ (see for instance [10], Theorem 2.2.1).

Let us illustrate the theorem with an example.

Example 4.10. Let $P=\mathbb{Q}[x, y]$. Consider the grid ideal

$$
J=\left\langle x\left(x^{2}+1\right)^{2}(x-1),\left(y^{3}-1\right)(y+2)\right\rangle
$$

with primary decomposition

$$
\begin{gathered}
\langle x, y+2\rangle \cap\langle x, y-1\rangle \cap\left\langle x, y^{2}+y+1\right\rangle \cap\langle x-1, y+2\rangle \cap\langle x-1, y-1\rangle \cap\left\langle x-1, y^{2}+y+1\right\rangle \\
\cap\left\langle x^{4}+2 x^{2}+1, y+2\right\rangle \cap\left\langle x^{4}+2 x^{2}+1, y-1\right\rangle \cap\left\langle x^{4}+2 x^{2}+1, y^{2}+y+1\right\rangle .
\end{gathered}
$$

Let $I_{1}$ and $I_{2}$ are complementary with respect to $J$. If

$$
I_{1}=\langle x, y+2\rangle \cap\left\langle x-1, y^{2}+y+1\right\rangle \cap\left\langle x^{4}+2 x^{2}+1, y+2\right\rangle,
$$

then

$$
\begin{gathered}
I_{2}=J: I_{1}=\langle x, y-1\rangle \cap\left\langle x, y^{2}+y+1\right\rangle \cap\langle x-1, y+2\rangle \cap\langle x-1, y-1\rangle \cap \\
\left\langle x^{4}+2 x^{2}+1, y-1\right\rangle \cap\left\langle x^{4}+2 x^{2}+1, y^{2}+y+1\right\rangle .
\end{gathered}
$$

We have $\operatorname{GFNum}\left(I_{1}\right)=\operatorname{GFNum}\left(I_{2}\right)=2$. See the following CoCoA-5 code for details. 


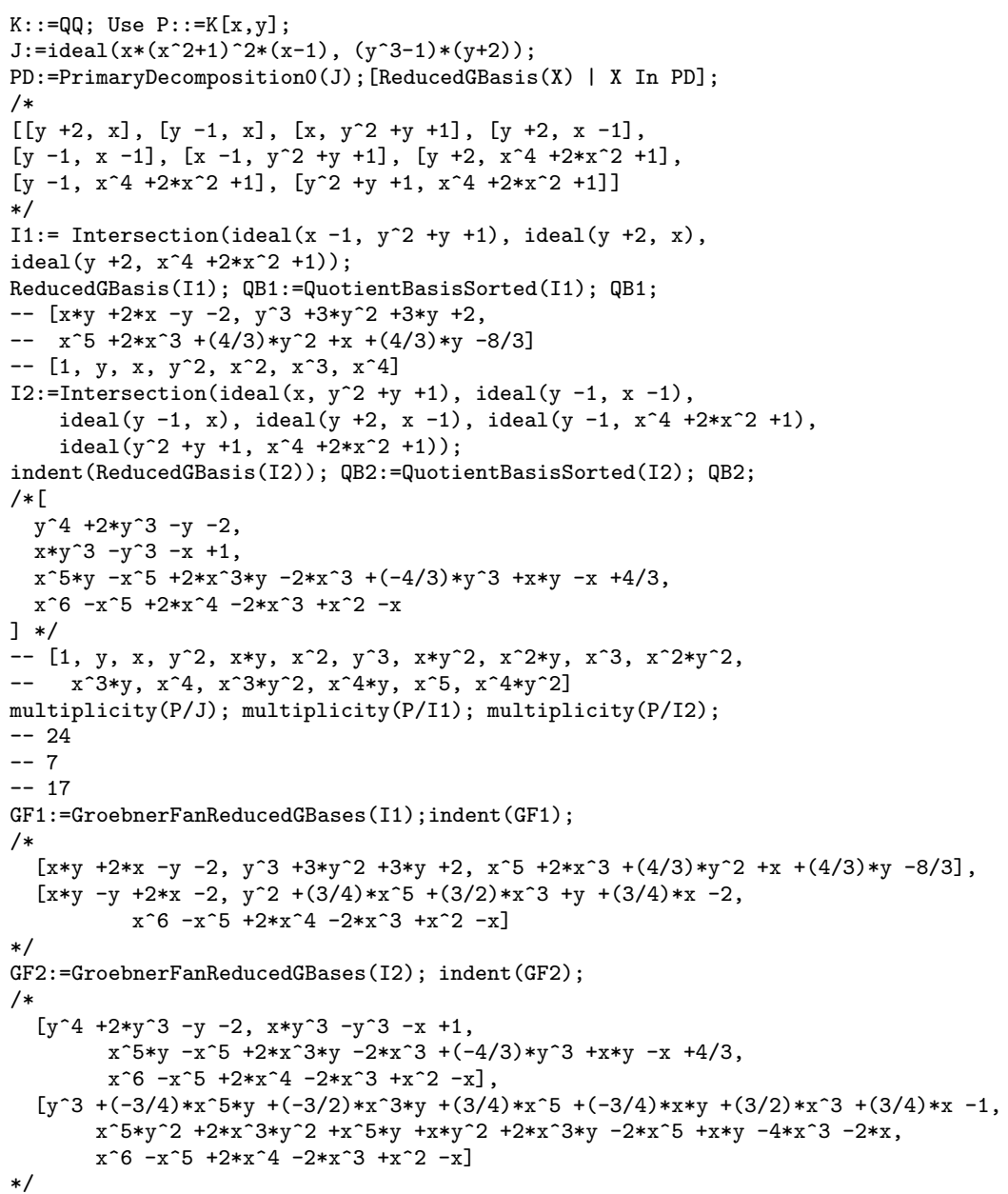

Corollary 4.11. Let $J$ be a radical grid ideal in $P$ and let $I$ be an ideal in $P$ such that $I \supseteq J$.

(a) The ideals $I$ and $J: I$ are radical.

(b) The ideals $I$ and $J: I$ are complementary ideals with respect to $J$, and hence the conclusions of Theorem 4.8 apply to $I_{1}=I$ and $I_{2}=J: I$.

(c) Let $\mathbb{X}$ be a grid of points, $J=\mathcal{I}(\mathbb{X})$, and $I \supseteq J$. Then there exists a subset $\mathbb{Y}$ of $\mathbb{X}$ such that $I=\mathcal{I}(\mathbb{Y}), J: I=\mathcal{I}(\mathbb{X} \backslash \mathbb{Y})$, and (a) and (b) are satisfied by the ideals $J, I$ and $J: I$.

Proof. To prove Claim (a), notice that as the ideal $J$ is zero-dimensional and radical, there are maximal ideals $\mathfrak{m}_{1}, \ldots, \mathfrak{m}_{s}$ in $P$ such that $J=\bigcap_{i=1}^{s} \mathfrak{m}_{i}$. Let $S=\{1, \ldots, s\}$. The Chinese Remainder Theorem implies that there is an isomorphism $\varphi: P / J \cong \prod_{i \in S} P / \mathfrak{m}_{i}$. Via this isomorphism, the image $\varphi(I)$ is 
a product of $s$ ideals which are either $\langle 0\rangle$ or $\langle 1\rangle$. Let $T \subseteq S$ be the subset of indices which correspond to the zero ideals. Then $I=\bigcap_{i \in T} \mathfrak{m}_{i}$ and hence it is radical. Similarly we find that $J: I$.

To prove Claim (b), it suffices to observe that we have $J: I=\bigcap_{i \in S \backslash T} \mathfrak{m}_{i}$.

Finally, to prove (c), let $\mathbb{X}$ be a grid of points in $K^{n}$, and for $i=1, \ldots, n$, let $g_{i}=\prod_{j=1}^{d_{i}}\left(x_{i}-c_{i j}\right)$. Then the vanishing ideal of $\mathbb{X}$ is $\mathcal{I}(\mathbb{X})=\left\langle g_{1}, \ldots, g_{n}\right\rangle$. The ideal $\mathcal{I}(\mathbb{X})$ is radical by construction, and every ideal which contains $\mathcal{I}(\mathbb{X})$ is the vanishing ideal of a subset $\mathbb{Y}$ of $\mathbb{X}$, i.e. it is of type $\mathcal{I}(\mathbb{Y})$. Consequently we have $\mathcal{I}(\mathbb{X}): \mathcal{I}(\mathbb{Y})=\mathcal{I}(\mathbb{X} \backslash \mathbb{Y})$.

The following example illustrates this corollary.

Example 4.12. $\mathrm{K}::=\mathrm{QQ}$; Use $\mathrm{P}: \mathrm{:}=\mathrm{K}[\mathrm{x}, \mathrm{y}]$;

$I:=$ ideal $\left(\left(\mathrm{x}^{\wedge} 2+1\right) *(\mathrm{x}-1) *(\mathrm{x}-2),\left(\mathrm{y}^{\wedge} 2-2\right) *(\mathrm{y}+2)\right)$;

$\mathrm{J} 1:=\mathrm{I}+$ ideal $\left(\mathrm{x}-1+\mathrm{y}^{\wedge} 2-2\right)$;

$\mathrm{J} 2:=\operatorname{Colon}(\mathrm{I}, \mathrm{J} 1)$;

ReducedGBasis (J1); QB1:=QuotientBasis (J1); QB1;

$-\left[\mathrm{x}-1, \mathrm{y}^{\wedge} 2-2\right]$

$--[1, y]$

ReducedGBasis(J2); QB2:=QuotientBasis(J2); QB2;

-- $\left[\mathrm{y}^{\wedge} 3+2 * \mathrm{y}^{\wedge} 2-2 * \mathrm{y}-4\right.$,

$\mathrm{x}^{\wedge} 3 * \mathrm{y}+2 * \mathrm{x}^{\wedge} 3-2 * \mathrm{x}^{\wedge} 2 * \mathrm{y}-4 * \mathrm{x}^{\wedge} 2+\mathrm{x} * \mathrm{y}+2 * \mathrm{x}-2 * \mathrm{y}-4$

$\left.\mathrm{x}^{\wedge} 4-3 * \mathrm{x}^{\wedge} 3+3 * \mathrm{x}^{\wedge} 2-3 * \mathrm{x}+2\right]$

-- $\left[1, y, y^{\wedge} 2, x, x * y, x * y^{\wedge} 2, x^{\wedge} 2, x^{\wedge} 2 * y, x^{\wedge} 2 * y^{\wedge} 2, x^{\wedge} 3\right]$

multiplicity(P/I); multiplicity(P/J1); multiplicity(P/J2);

$--12$

$--2$

$--10$

GF1:=GroebnerFanReducedGBases (J1);GF1;

$--\left[y^{\wedge} 2-2, x-1\right]$

GF2: =GroebnerFanReducedGBases (J2) ; GF2 ;

-- $\left[\mathrm{y}^{\wedge} 3+2 * \mathrm{y}^{\wedge} 2-2 * \mathrm{y}-4\right.$,

-- $\mathrm{x}^{\wedge} 3 * \mathrm{y}+2 * \mathrm{x}^{\wedge} 3-2 * \mathrm{x}^{\wedge} 2 * \mathrm{y}-4 * \mathrm{x}^{\wedge} 2+\mathrm{x} * \mathrm{y}+2 * \mathrm{x}-2 * \mathrm{y}-4$,

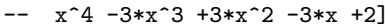

\section{Final Remarks}

In this section we collect some consequences of the theoretical results described in the preceding sections. The significance of Theorem 4.8 is the ability to quickly compute new ideals with GFNum 1 . This is especially convenient for network inference or design of experiments where data often have states in a finite field. Let $K$ be a finite field with characteristic $p>0$. Then $K$ is a finitedimensional $\mathbb{F}_{p}$-vector space hence the number of its elements is $q=p^{e}$, where $e=\operatorname{dim}_{\mathbb{F}_{p}}(K)$. Given an indeterminate $z$, the univariate polynomial $z^{q}-z$ is called a field equation of $K$ since $z^{q}-z=\prod_{a \in K}(z-a)$ (see [7], Section 4.13). Consequently, if $P=K\left[x_{1}, \ldots, x_{n}\right]$ and $g_{i}=x_{i}^{q}-x_{i}$ for $i=1, \ldots, n$, then the ideal $\left\langle g_{1}, \ldots, g_{n}\right\rangle$ is the vanishing ideal of a grid and hence Corollary 4.11 applies to this case.

Let us see an example with $K=\mathbb{F}_{3}$. 
Example 5.1. $K::=z Z /(3)$; Use $P::=K[x, y, z]$;

I:=ideal $\left(x^{\wedge} 3-x, y \wedge 3-y, z^{\wedge} 3-z\right)$;

$\mathrm{J} 1:=\mathrm{I}+\mathrm{ideal}\left(\mathrm{x}^{\wedge} 2-\mathrm{y}-\mathrm{z}\right)$;

$\mathrm{J} 2:=\operatorname{Colon}(\mathrm{I}, \mathrm{J} 1)$;

ReducedGBasis (J1); QB1:=QuotientBasis (J1); QB1;

-- $\left[y^{\wedge} 2-y * z+z \wedge 2-y-z, x * y+x * z-x, x \wedge 2-y-z, z^{\wedge} 3-z\right]$

$--\left[1, z, z^{\wedge} 2, y, y * z, y * z^{\wedge} 2, x, x * z, x * z^{\wedge} 2\right]$

ReducedGBasis (J2); QB2:=QuotientBasis(J2); QB2;

$-\left[\mathrm{z}^{\wedge} 3-\mathrm{z}, \mathrm{y}^{\wedge} 3-\mathrm{y}, \mathrm{x} * \mathrm{y} \wedge 2-\mathrm{x} * \mathrm{y} * \mathrm{z}+\mathrm{x} * \mathrm{z} \wedge 2+\mathrm{x} * \mathrm{y}+\mathrm{x} * \mathrm{z}\right.$,

$\left.x^{\wedge} 2 * y+x^{\wedge} 2 * z+x^{\wedge} 2+y^{\wedge} 2-y * z+z^{\wedge} 2-1, x \wedge 3-x\right]$

-- $\left[1, \mathrm{z}, \mathrm{z} \mathrm{z}^{\wedge} 2, \mathrm{y}, \mathrm{y} * \mathrm{z}, \mathrm{y} * \mathrm{z}^{\wedge} 2, \mathrm{y}^{\wedge} 2, \mathrm{y}^{\wedge} 2 * \mathrm{z}, \mathrm{y}^{\wedge} 2 * \mathrm{z}^{\wedge} 2, \mathrm{x}, \mathrm{x} * \mathrm{z}\right.$, $\left.x * z^{\wedge} 2, x * y, x * y * z, x * y * z^{\wedge} 2, x \wedge 2, x \wedge 2 * z, x \wedge 2 * z^{\wedge} 2\right]$

multiplicity(P/I); multiplicity(P/J1); multiplicity(P/J2);

$--27$

$--9$

$--18$

GF $1:=$ GroebnerFanIdeals (J1);

GF2:=GroebnerFanIdeals (J2);

Len (GF1); Len (GF2);

$--4$

$--4$

GF1 : $=$ GroebnerFanReducedGBases $(\mathrm{J} 1)$; indent (GF1);

/*

[

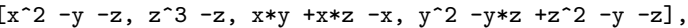

$\left[x^{\wedge} 2-z-y, y^{\wedge} 3-y, x * z+x * y-x, z^{\wedge} 2-y * z+y \wedge 2-z-y\right]$,

$\left[y-x^{\wedge} 2+z, x \wedge 3-x, \quad z \wedge 3-z\right]$

$\left[z+y-x^{\wedge} 2, x \wedge 3-x, y \wedge 3-y\right]$

*/

GF2:=(J2); indent (GF2);

*

$\left[\mathrm{z}^{\wedge} 3-\mathrm{z}, \mathrm{y} \wedge 3-\mathrm{y}, \mathrm{x} * \mathrm{y} \wedge 2-\mathrm{x} * \mathrm{y} * \mathrm{z}+\mathrm{x} * \mathrm{z} \wedge 2+\mathrm{x} * \mathrm{y}+\mathrm{x} * \mathrm{z}\right.$,

$\left.\mathrm{x}^{\wedge} 2 * \mathrm{y}+\mathrm{x}^{\wedge} 2 * \mathrm{z}+\mathrm{x}^{\wedge} 2+\mathrm{y} \wedge 2-\mathrm{y} * \mathrm{z}+\mathrm{z}^{\wedge} 2-1, \mathrm{x}^{\wedge} 3-\mathrm{x}\right]$,

$\left[y^{\wedge} 3-y, x^{\wedge} 3-x, x^{\wedge} 2 * z+x^{\wedge} 2 * y+z^{\wedge} 2+x^{\wedge} 2-y^{*} * z+y \wedge 2-1\right.$, $\mathrm{x} * \mathrm{z} \wedge 2-\mathrm{x} * \mathrm{y} * \mathrm{z}+\mathrm{x} * \mathrm{y} \wedge 2+\mathrm{x} * \mathrm{z}+\mathrm{x} * \mathrm{y}, \mathrm{z} \wedge 3-\mathrm{z}$ ],

$\left[x^{\wedge} 3-x, z^{\wedge} 3-z, y^{\wedge} 2+x^{\wedge} 2 * y-y * z+x^{\wedge} 2 * z+z^{\wedge} 2+x^{\wedge} 2-1\right]$, $* 1$

$\left[x^{\wedge} 3-x, \quad z^{\wedge} 2-y * z+y^{\wedge} 2+x \wedge 2 * z+x^{\wedge} 2 * y+x^{\wedge} 2-1, y^{\wedge} 3-y\right]$

Theorem 4.8 shows, among other results, that complementary ideals have the same number of reduced Gröbner bases. The advantage of this is that it may be computationally easy to test whether a small set of data has a unique Gröbner basis associated to it and then to generate a larger set via the complement. Let us see an easy application of this remark.

Proposition 5.2. Let $J, I_{1}, I_{2}$ be deals in $P$ such that $J$ and $I_{1}$ are grid ideals, $J \subset I_{1}$, and $I_{2}=J: I_{1}$,

(a) We have $\operatorname{GFNum}\left(I_{2}\right)=1$.

(b) In particular, statement (a) holds if $\mathbb{X}, \mathbb{Y}$ are grid of points, $J=\mathcal{I}(\mathbb{X})$, $I_{1}=\mathcal{I}(\mathbb{Y})$, and hence $I_{2}=\mathcal{I}(\mathbb{X} \backslash \mathbb{Y})$.

Proof. As Claim (b) is a special case of (a), let us prove Claim (a). Since $I_{2}$ is a grid ideal, we get $\operatorname{GFNum}\left(I_{2}\right)=1$ from Lemma 4.5(b), and the conclusion follows from Theorem $4.8(\mathrm{~d})$.

Let us see an example which illustrates this proposition. 
Example 5.3. Use $P::=Q Q[x, y]$;

$\mathrm{F}:=\mathrm{x} *(\mathrm{x}-1) *(\mathrm{x}-2) *(\mathrm{x}-3) *(\mathrm{x}-4)$;

$\mathrm{G}:=\mathrm{y} *(\mathrm{y}-1) *(\mathrm{y}-2) *(\mathrm{y}-3)$;

$I:=i d e a l(F, G)$;

$M:=\operatorname{mat}([[0,1],[0,3],[1,1],[1,3],[3,1],[3,3]])$;

$\mathrm{J} 1:=$ Idealof Points $(\mathrm{P}, \mathrm{M})$;

$\mathrm{J} 2:=\operatorname{Colon}(\mathrm{I}, \mathrm{J} 1)$;

GF : $=$ GroebnerFanIdeals $(\mathrm{J} 2) ; \mathrm{GF}$;

-- [ideal $\left(\mathrm{x}^{\wedge} 2 * \mathrm{y}^{\wedge} 2-2 * \mathrm{x}^{\wedge} 2 * \mathrm{y}-6 * \mathrm{x} * \mathrm{y} \wedge 2+12 * \mathrm{x} * \mathrm{y}+8 * \mathrm{y} \wedge 2-16 * \mathrm{y}\right.$,

-- $\left.\left.\mathrm{y}^{\wedge} 4-6 * \mathrm{y}^{\wedge} 3+11 * \mathrm{y}^{\wedge} 2-6 * \mathrm{y}, \mathrm{x}^{\wedge} 5-10 * \mathrm{x}^{\wedge} 4+35 * \mathrm{x}^{\wedge} 3-50 * \mathrm{x}^{\wedge} 2+24 * \mathrm{x}\right)\right]$

Len (GF);

$--1$

-- The ideal $\mathrm{J} 1$ is the vanishing ideal of the "white dots".

-- The ideal J2 is the vanishing ideal of the "black dots".

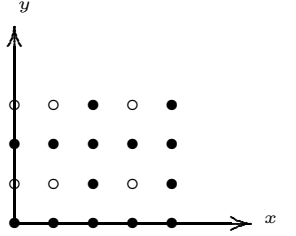

One of the main goals of this paper is to identify classes of ideals inside affine $K$-algebras which have a GFan number equal to 1 . Using the notions of distractions of ideals and their linear shifts we were able to identify a large class of such ideals and provided a methodology for constructing them. Furthermore, we proved that complementary ideals have the same GFan number which provides a tool for identifying ideals of (large) sets of points as having a GFan number 1 based on the ideal of the (small) complementary set of points. Future work may involve a geometric characterization of all data sets with GFan number equal to 1 .

\section{Acknowledgements}

We thank Shuhong Gao and Sean Sather-Wagstaff for their insightful comments during fruitful discussions. We also thank Anyu Zhang for the helpful computations she performed. Dimitrova, He, and Stigler were partially supported by the National Science Foundation under Award DMS-1419023. Finally, we are pleased to thank the anonymous referee for many useful comments.

\section{References}

\section{References}

[1] J. Abbott, A. M. Bigatti, CoCoA-5: A system for doing Computations in Commutative Algebra, available at http://cocoa.dima.unige.it.

[2] J. Abbott, A. Bigatti, M. Kreuzer, L. Robbiano, Computing Ideals of Points, J. Symb. Comput. 30 (4), (2000), 341-356. 
[3] J. Abbott, M. Kreuzer, L. Robbiano, Computing zero-dimensional Schemes, J. Symb. Comput. 39 (2005), 31-49.

[4] E. Babson, S. Onn, R. Thomas, The Hilbert Zonotope and a polynomial time algorithm for universal Gröbner bases, Adv. Appl. Math. 30, (2003), $529-544$.

[5] E.S. Dimitrova, B. Stigler, Data Identification for Improving Gene Network Inference using Computational Algebra, Bull. Math. Biol. 76(11), (2014), $2923-2940$.

[6] R. Garcia, L.D. García Puente, R. Kruse, J. Liu, D. Miyata, E. Petersen, K. Phillipson, A Shiu, Gröbner Bases of Neural Ideals, International Journal of Algebra and Computation. 28(4), (2018), 553-571.

[7] N. Jacobson, Basic Algebra I, 2nd ed., Dover Publications, Mineola (2009).

[8] M. Kreuzer, L. Robbiano, Computational Commutative Algebra 1, 2nd ed., Springer (2008).

[9] M. Kreuzer, L. Robbiano, Computational Commutative Algebra 2, Springer (2005).

[10] M. Kreuzer, L. Robbiano, Computational Linear and Commutative Algebra, Springer (2016).

[11] R. Laubenbacher, B. Stigler, A computational algebra approach to the reverse engineering of gene regulatory networks, J. Theor. Biol. 229(4), (2004), 523-537.

[12] Z. Lin, L. Xu, Q. Wu, Applications of Gröbner bases to signal and image processing: A survey, Linear Algebra Appl. 391, (2004), 169-202.

[13] M. Maniatis, A. von Manteuffel, O. Nachtmann, Determining the global minimum of Higgs potentials via Gröbner bases Applied to the NMSSM, The European Physical Journal 49(4), (2007), 1067-1076.

[14] T. Mora, L. Robbiano, The Gröbner Fan of an Ideal, J. Symb. Comput. 6, (1988), 183-208.

[15] L. Robbiano, Gröbner Bases and Statistics, In "Gröbner Bases and Applications" Proceedings of the Conference "33 Years of Gröbner Bases" (1998). London Mathematical Society, Lecture Notes Series Vol 251, B. Buchberger and F. Winkler eds. Cambridge University Press, 179-204.

[16] M. Torrente, Applications of Algebra in the Oil Industry, Scuola Normale Superiore di Pisa, (2009).

[17] Y.-L. Tsai, Estimating the number of tetrahedra determined by volume, circumradius and four face areas using Groebner basis, J. Symb. Comput. 77, (2006), 162-174. 